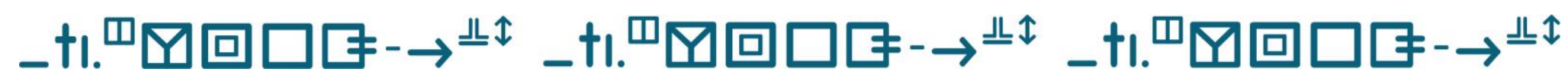

\section{Senso de autoeficácia de professores de alunos com autismo surdos em escolas bilíngues}

\section{The self-efficacy sense of the teachers of deaf autistic students in bilingual schools}

\footnotetext{
iD (9) Raquel Aparecida Lopes

INSPER - Instituto de Ensino e Pesquisa, São Paulo, Brasil stampa12@uol.com

(D) Cibelle Albuquerque de La Higuera Amato

Universidade Presbiteriana Mackenzie, São Paulo, Brasil cibelle.amato@mackenzie.br

iD 9

Maria Cecília de Moura

Pontifícia Universidade Católica de São Paulo, São Paulo, Brasil alce55@uol.com.br

Resumo: A Autism Self-Efficacy Scale for Teachers (ASSET) avalia as crenças de autoeficácia dos professores em sua capacidade para desempenhar tarefas no ensino de alunos com Transtorno do Espectro do Autismo (TEA). O estudo objetivou identificar e analisar a autoeficácia docente (AED) de 38 professores de alunos com TEA surdos atuantes em EMEBS - Escolas Municipais de Educação Bilíngue para surdos e em escolas regulares polos de atendimento inclusivos e bilíngues para surdos no Brasil. Como instrumento de coleta de dados, utilizou-se a ASSET. Os resultados apontam que a maioria dos participantes está confiante com os itens avaliados, o que pode ser um potencial preditor das atitudes 


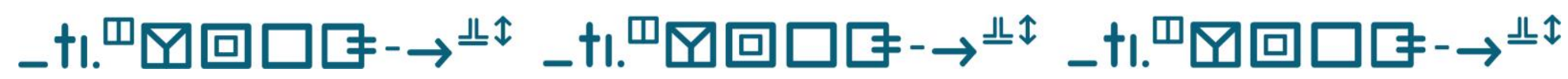

docentes em contextos educacionais bilíngues, uma vez que a autoeficácia exerce um papel determinante do pensamento, comportamento e desempenho de uma ação. Este trabalho ressalta a importância de instrumentos de autoavaliação voltados à professores que atuam na educação pessoas com TEA surdas no Brasil.

Palavras-chave: Autoeficácia docente. Transtorno do Espectro Autista. Surdos.

Abstract: The Autism Self-Efficacy Scale for Teachers (ASSET) assesses teachers' self-efficacy beliefs in their ability to perform tasks in teaching students with Autism Spectrum Disorder (ASD). The study aimed to identify and analyze the self-efficacy belief (AED) of 38 teachers of deaf students with ASD working in EMEBS - Municipal Schools of Bilingual Education for the Deaf - and in regular schools for inclusive and bilingual care centers for the deaf in Brazil. As a data collection instrument, ASSET was used. The results show that the majority of participants are confident with the items evaluated, which can be a potential predictor of teaching attitudes in bilingual educational contexts, since self-efficacy plays a determining role in the thinking, behavior and performance of an action. This work highlights the importance of self-assessment instruments aimed at teachers who work in education for people with deaf ASD in Brazil.

Keywords: Deaf Literature. Creation. Adaptation. Translation.

Submetido em 08 de março de 2021.

Aceito em 21 de maio de 2021.

Publicado em 19 de novembro de 2021. 


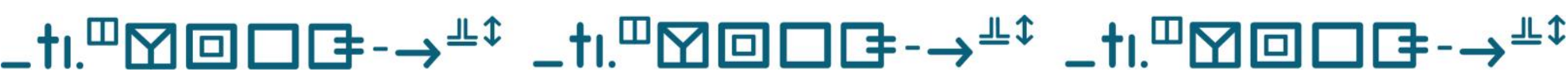

Senso de autoeficácia de professores de alunos com autismo surdos em escolas bilíngues Raquel Aparecida Lopes • Cibelle Albuquerque de La Higuera Amato et al.

\section{Introdução}

A ideia de que os indivíduos agem de acordo com suas próprias crenças está diretamente relacionada ao conceito de autoeficácia, que é definida como o julgamento que ele faz sobre suas habilidades e capacidades para atuar em um domínio específico, constituindo-se em alicerce para a motivação, o bem-estar e as futuras realizações pessoais (BANDURA, 1986, 1993, 1997). A forma de agir de acordo com as suas autocrenças Ihes permite assumir um determinado grau de controle sobre seus sentimentos, pensamentos e ações. Assim, ao optar por realizar uma certa tarefa, o indivíduo também faz uma análise de suas habilidades e capacidades atuais, julgando se será ou não capaz de atingir seus objetivos na tomada de decisões para alcançá-los (PAJARES; OLAZ, 2008).

Os estudos sobre autoeficácia no Brasil se concentram na área da saúde e educação e apresentam evidências relacionadas à eficácia coletiva de professores. Assim, compreende-se que crenças educacionais são percepções e ideias ligadas à temas do contexto educacional, os quais se mostram, consciente ou inconscientemente, nas ações dos professores (BZUNECK; GUIMARÃES, 2003; AZZl; POLYDORO, 2006). Certamente essas crenças acabam por influenciar nas tomadas de decisões pedagógicas, causando impacto direto no processo de ensino-aprendizagem, uma vez que o professor, diante de dificuldades ou facilidades, pode criar diferentes expectativas em relação aos alunos, inclusive com alunos que possuem alguma Necessidade Educacional Especial (NEE).

De acordo com Canabarro, Teixeira e Schmidt (2018), as escalas Teacher Efficacy Scale (TES), de Gibson e Dembo (1984) e Teacher Interpersonal Self-Efficacy Scale (TISES), de Brouwers e Tomic (2001), são utilizadas para avaliar a autoeficácia docente. Contudo, o estudo de Klassen et al. (2011) 


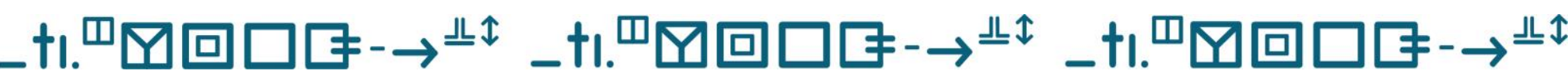

Senso de autoeficácia de professores de alunos com autismo surdos em escolas bilíngues Raquel Aparecida Lopes • Cibelle Albuquerque de La Higuera Amato et al.

assinala que esses instrumentos de medidas gerais de autoeficácia não compreendem a avaliação de crenças dos professores que atuam com alunos com necessidades educacionais especiais. No Brasil, o público-alvo que abrange a Educação Especial constitui-se por "alunos com deficiências sensoriais, deficiência mental/intelectual, transtornos globais do desenvolvimento/ Transtorno do Espectro Autista (TEA), deficiências múltiplas (associação de duas ou mais deficiências primárias) e altas habilidades (superdotados)" (CANABARRO; TEIXEIRA; SCHMIDT, 2018, p. 231). Outras condições que podem gerar prejuízos de aprendizagem podem não estar relacionadas necessariamente à alguma deficiência, uma vez que podem se apresentar também em alunos com problemas de atenção e/ou hiperatividade, dislexia, entre outros transtornos do neurodesenvolvimento (BRASIL, 2008; CANABARRO; TEIXEIRA; SCHMIDT, 2018).

Dentre as condições mencionadas, o TEA está entre os mais graves e é caracterizado por um quadro clínico em que prevalecem prejuízos na comunicação social, interação social e comportamento, causando frequentemente comprometimento do funcionamento adaptativo, sobretudo, no ambiente escolar. Estes prejuízos podem se apresentar em graus variáveis, além de déficits persistentes nas interações sociais, padrões restritos e repetitivos de comportamento, interesses ou atividade (ARAÚJO, 2011; NUNES; AZEVEDO; SCHMIDT, 2013; APA, 2014).

No que se refere à questão do indivíduo surdo, observa-se que tem sido extensivamente estudada nos últimos anos (HARRISON; MOURA, 2013; GARRUTI-LOURENÇO, 2017; CAMPOS, 2017; MOURA, 2018; LOPES, 2020). Temas relacionados ao desenvolvimento de linguagem, identidade, cultura e comunidade surda, assim como àqueles ligados ao processo educacional são motivo de preocupação e de pesquisas que apontam a importância da Libras (Língua Brasileira de Sinais) em todos esses aspectos acima mencionados. 


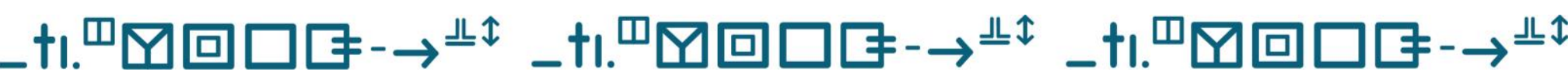

Senso de autoeficácia de professores de alunos com autismo surdos em escolas bilíngues Raquel Aparecida Lopes • Cibelle Albuquerque de La Higuera Amato et al.

Compreender como pode se dar a inclusão de surdos no ensino regular ou como a escola bilíngue para surdos pode cumprir suas funções educacionais é um desafio que ainda tem um grande caminho pela frente.

O surdo/deficiente auditivo não é percebido pela falta de audição na sociedade, mas pelas necessidades específicas com relação à linguagem e a sua pertinência ao grupo social que fala uma língua nem sempre compreendida por ele. Outro aspecto que merece consideração está relacionado ao seu desempenho acadêmico. O surdo/deficiente auditivo tem necessidades educacionais específicas que estão intimamente ligadas à sua forma de estar no mundo, isto é, a forma visual de receber informações e perceber o mundo que the circunda, mesmo quando faz uso de amplificação sonora ou de implante coclear (MOURA, 2000).

Quando se dirige o olhar para o indivíduo autista surdo, a situação tende a se tornar mais complexa. Afinal, como um indivíduo que depende da visão para poder se relacionar com o mundo externo (por ser surdo/deficiente auditivo) e que tem como uma das suas dificuldades essenciais a falha ou ausência de contato visual (por estar no espectro autista) (LUZ; GOMES; LIRA, 2017) poderá entrar em contato com o outro que Ihe deve fornecer informações que têm um cunho altamente visual? Em se tratando de relacionar-se com o mundo externo, poderíamos nos indagar, ainda, sobre como as estratégias utilizadas pelos professores podem contribuir para que este indivíduo consiga se comunicar?

Sob este ponto, estudos pioneiros no Brasil como o de Lopes (2020) e Lopes e Amato (2021) têm apontado para a importância do uso de diferentes estratégias de comunicação, uma vez que, pela presença da surdez, é necessário também reconhecê-lo como sujeito visual, que interage com o conhecimento a partir de suas experiências visuais, mesmo que o contato visual reduzido seja um sinal clássico no autismo. Ainda que essa condição 


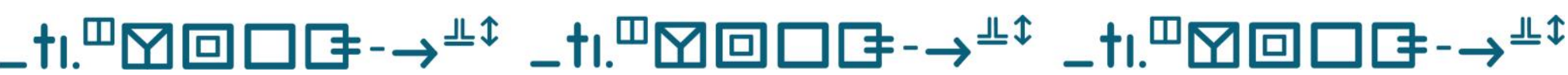

Senso de autoeficácia de professores de alunos com autismo surdos em escolas bilíngues Raquel Aparecida Lopes • Cibelle Albuquerque de La Higuera Amato et al.

ocasione prejuízos, esse aluno deve ser auxiliado pelos sistemas de Comunicação Alternativa e Ampliada (CAA) no ambiente escolar, conforme explicam Lopes e Amato (2021, p. 112):

\begin{abstract}
A American Speech and Hearing association define a CAA como recursos, símbolos, estratégias e técnicas utilizadas pelos indivíduos para complementar a comunicação. Estes sistemas podem ser divididos de acordo com o tipo de uso de instrumento, em: com auxílio e sem auxílio instrumental. Dentre os que utilizam auxílio instrumental, são amplamente conhecidos o PECS ${ }^{1}$ e o TEACCH ${ }^{2}$, as línguas de sinais ${ }^{3}$ são utilizadas para melhora das habilidades comunicativas e não necessitam de auxílio instrumental. É importante esclarecer que as línguas de sinais são línguas naturais de uma população sem déficits cognitivos da espécie humana, o surdo.
\end{abstract}

Estes estudos evidenciam que a Libras vem sendo utilizada como principal estratégia de comunicação de autistas surdos nas EMEBS - Escolas Municipais de Educação Bilíngue para Surdos e em escolas regulares polos de atendimento inclusivos e bilíngues para surdos, apesar dos professores considerarem que estes alunos apresentam uma evolução diferente em relação aos surdos na aquisição dos sinais. Para as pesquisadoras, isso ocorre pelos prejuízos relacionados ao TEA e à ausência da audição, e faz com que essa condição na escola por vezes se agrave, pois contribui para que eles se encontrem mais isolados, o que ressalta a importância de ações educativas que visem o avanço de suas habilidades.

Os estudos de Fulwiler e Fouts (1976), Carr et al. (1978) e Stull et al. (1979) apontam para o uso da Língua de Sinais como estratégia de comunicação para pessoas com TEA surdas e, apesar da escassez de investigações científicas no Brasil neste campo do conhecimento, em outros países esses estudos já são realizados, sobretudo, nos Estados Unidos. As pesquisadoras relatam que foi no final dos anos 70 e começo dos anos 80 


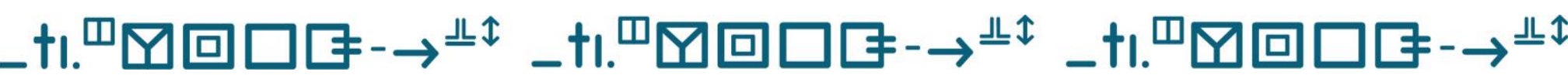

Senso de autoeficácia de professores de alunos com autismo surdos em escolas bilíngues Raquel Aparecida Lopes • Cibelle Albuquerque de La Higuera Amato et al.

que a Língua de Sinais começou a ser vista como uma possibilidade de comunicação alternativa para crianças com autismo. Segundo Stull et al. (1979), as pesquisas pertinentes ao aprendizado da língua concentraram-se em crianças com audição e minimamente verbal com forma severa de TEA.

Lopes e Amato (2021) assinalam o primeiro estudo realizado sobre o uso de pronomes, com uma população pouco estudada, de crianças com TEA surdas expostas à Língua de Sinais Americana (ASL) desde o nascimento por pais surdos, em que:

[...] dois grupos de participantes foram testados: (1) crianças com TEA surdas que utilizam a língua de sinais e (2) crianças surdas com desenvolvimento típico. As evidências encontradas, por meio de uma tarefa de evocação e relato parental, são de que as crianças com TEA surdas que utilizam língua de sinais evitam pronomes em favor de nomes. Uma análise de uso espontâneo mostrou que todas as crianças demonstraram habilidade de apontar, mas apenas crianças com melhor capacidade de utilizar a língua de sinais produziram pronomes. Para o pesquisador é comum crianças com TEA verbais inverterem os pronomes, referindose a si próprios como "ele" especialmente os mais novos, sendo que essas inversões também ocorrem com crianças com TEA surdas em língua de sinais (SHIELD, MEIER e FLUSBERG, 2015, p. 4 apud LOPES e AMATO, 2021, p. 121).

Estes resultados permitem dizer que o trabalho com alunos com necessidades educacionais especiais, como por exemplo o autismo e a surdez, exige do professor conhecimentos específicos e envolvem acomodações e flexibilizações no currículo escolar, que podem colaborar ou não para crenças adequadas de autoeficácia. Desta forma, optou-se por utilizar a ASSET neste estudo, pois escalas que permitem identificar o senso de autoeficácia de professores na atuação profissional junto à alunos com TEA “podem ser importantes instrumentos para auxiliar no reconhecimento e autoavaliação do processo de ensino e aprendizagem" (CANABARRO; 


\section{_tı.}

Senso de autoeficácia de professores de alunos com autismo surdos em escolas bilíngues Raquel Aparecida Lopes • Cibelle Albuquerque de La Higuera Amato et al.

TEIXEIRA; SCHMIDT, 2018, p. 231). Esses autores apontam que, dentre as escalas desenvolvidas para avaliação de autoeficácia, apenas a Autism SelfEfficacy Scale for Teachers (ASSET) (RUBLE et al., 2013) avalia o domínio específico para a atuação de professores dessa população. A escala foi desenvolvida nos Estados Unidos e avaliada com resultados positivos em grupo amostral composto por 44 professores de Educação Especial em escolas de dois estados do centro-sul dos EUA.

O objetivo central deste estudo foi identificar e analisar o senso de autoeficácia de professores que atuam com alunos com TEA surdos.

\section{Método}

O estudo teve um desenho do tipo survey descritivo, utilizou-se a Autism Self-Efficacy Scale for Teachers (ASSET) como instrumento de coleta de dados que avalia as crenças de autoeficácia dos professores em sua capacidade para desempenhar tarefas docentes no ensino de alunos com autismo.

A ASSET foi traduzida, validada e publicada em português e permite que o professor autoavalie sua confiança numa escala do tipo likert que vai de 0 (não posso fazer nada) a 5 (certamente posso fazer). O Quadro 1 mostra a escala ASSET, composta por 30 itens (questões) a respeito do quão confiante os professores se sentem em relação aos principais conhecimentos e habilidades necessárias ao atendimento do aluno com autismo. O questionário foi concebido para auxiliar a compreensão das situações e contextos que criam dificuldades para os professores de alunos com autismo (CANABARRO; TEIXEIRA; SCHMIDT, 2018). 


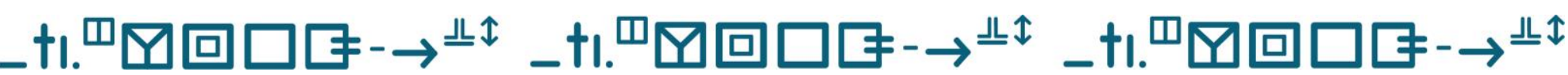

Senso de autoeficácia de professores de alunos com autismo surdos em escolas bilíngues Raquel Aparecida Lopes • Cibelle Albuquerque de La Higuera Amato et al.

\begin{tabular}{|l|l|l|}
\hline $\mathbf{1 8 .}$ & Avaliar as habilidades de interação social do aluno. \\
\hline $\mathbf{1 9}$. & Avaliar a habilidade de brincar do aluno. \\
\hline $\mathbf{2 0 .}$ & Ensinar habilidades/estratégias de interação social ao aluno. \\
\hline $\mathbf{2 1 .}$ & Ensinar ao aluno habilidades de brincar. \\
\hline $\mathbf{2 2 .}$ & Ensinar os colegas para aprimorar as habilidades sociais do aluno. \\
\hline $\mathbf{2 3 .}$ & Comunicar e trabalhar efetivamente com os pais ou cuidadores do aluno. \\
\hline $\mathbf{2 4 .}$ & Descrever prioridades dos pais em relação à aprendizagem do aluno. \\
\hline $\mathbf{2 5 .}$ & Auxiliar o aluno a manter-se engajado. \\
\hline $\mathbf{2 6 .}$ & Sustentar a atenção do aluno. \\
\hline $\mathbf{2 7 .}$ & Motivar o aluno. \\
\hline $\mathbf{2 8 .}$ & Auxiliar o aluno a sentir-se competente. \\
\hline $\mathbf{2 9 .}$ & Ensinar habilidades acadêmicas ao aluno. \\
\hline $\mathbf{3 0 .}$ & & \\
\hline
\end{tabular}

Fonte: Canabarro; Teixeira e Schmidt (2018, p. 243).

Descrição da imagem: Gráfico informativo sobre os itens que compõem a escala Autism SelfEfficacy Scale for Teachers (ASSET).

O instrumento foi aplicado em plataforma online com acesso pelo https://docs.google.com e os participantes assinaram o Termo de Consentimento Livre e Esclarecido na própria plataforma. A amostra seguiu critérios de conveniência e participaram do estudo 38 professores de alunos com TEA surdos. Optou-se por uma análise da frequência das respostas. O cálculo da frequência absoluta ( $n i)$ e da frequência relativa ( $f i)$ foi realizado por meio de software de planilhas eletrônicas. O estudo foi aprovado pelo Comitê de Ética em Pesquisa com Seres Humanos da Universidade Presbiteriana Mackenzie, sob o protocolo CAAE: 09409219.4.0000.0084.

Não existem escalas no Brasil que avaliam a autoeficácia no desempenho das funções de professor no que se refere ao atendimento de alunos com TEA surdos, por este motivo utilizamos a ASSET neste estudo, uma vez que a escala se mostrou um importante instrumento de autoavaliação para profissionais que atuam com alunos com TEA. 


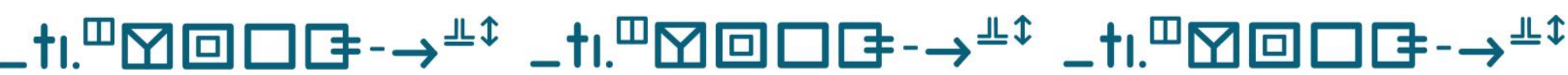

Senso de autoeficácia de professores de alunos com autismo surdos em escolas bilíngues Raquel Aparecida Lopes • Cibelle Albuquerque de La Higuera Amato et al.

\section{Resultados e Discussão}

Os dados obtidos com o preenchimento do instrumento foram tabulados e serão apresentados a seguir.

A Tabela 1 mostra a frequência de respostas ni (fi) para cada item (questão) apresentada na escala ASSET. As colunas de zero a cinco correspondem aos graus de confiança do professor, em que zero é a incapacidade de fazer e cinco é a certeza de possibilidade de realização.

Tabela 1 - Frequências absoluta e relativa das respostas à escala ASSET. ni (fi)

\begin{tabular}{|c|c|c|c|c|c|c|}
\hline Item & 0 & 1 & 2 & 3 & 4 & 5 \\
\hline 1 & $3(7,9 \%)$ & 7 18,4\%) & $2,3 \%)$ & 5 13,2\%) & $7(18,4 \%)$ & $14(36,8 \%)$ \\
\hline 2 & $1(2,6 \%)$ & $9(23,7 \%)$ & $0(0,0 \%)$ & $2(5,3 \%)$ & $6(15,8 \%)$ & $20(52,6 \%)$ \\
\hline 3 & $1(2,6 \%)$ & $8(21,1 \%)$ & $2(5,3 \%)$ & $5(13,2 \%)$ & $7(18,4 \%)$ & $15(39,5 \%)$ \\
\hline 4 & $2(5,3 \%)$ & $7(18,4 \%)$ & $0(0,0 \%)$ & $4(10,5 \%)$ & $12(31,6 \%)$ & $13(34,2 \%)$ \\
\hline 5 & $2(5,3 \%)$ & $7(18,4 \%)$ & $0(0,0 \%)$ & $6(15,8 \%)$ & $5(13,2 \%)$ & $18(47,4 \%)$ \\
\hline 6 & $3(7,9 \%)$ & $6(15,8 \%)$ & $0(0,0 \%)$ & $4(10,5 \%)$ & $4(10,5 \%)$ & $21(55,3 \%)$ \\
\hline 7 & $1(2,6 \%)$ & $8(21,1 \%)$ & $0(0,0 \%)$ & $5(13,2 \%)$ & $3(7,9 \%)$ & $21(55,3 \%)$ \\
\hline 8 & $2(5,3 \%)$ & $7(18,4 \%)$ & $0(0,0 \%)$ & $2(5,3 \%)$ & $7(18,4 \%)$ & $20(52,6 \%)$ \\
\hline 9 & $4(10,5 \%)$ & $7(18,4 \%)$ & $1(2,6 \%)$ & $2(5,3 \%)$ & $6(15,8 \%)$ & $18(47,4 \%)$ \\
\hline 10 & $5(13,2 \%)$ & $6(15,8 \%)$ & $3(7,9 \%)$ & $2(5,3 \%)$ & $5(13,2 \%)$ & $17(44,7 \%)$ \\
\hline 11 & $6(15,8 \%)$ & $6(15,8 \%)$ & $2(5,3 \%)$ & $3(7,9 \%)$ & $4(10,5 \%)$ & $17(44,7 \%)$ \\
\hline 12 & $5(13,2 \%)$ & $5(13,2 \%)$ & $2(5,3 \%)$ & $4(10,5 \%)$ & $5(13,2 \%)$ & $17(44,7 \%)$ \\
\hline 13 & $2(5,3 \%)$ & $7(18,4 \%)$ & $2(5,3 \%)$ & $2(5,3 \%)$ & $12(31,6 \%)$ & $13(34,2 \%)$ \\
\hline 14 & $4(10,5 \%)$ & $7(18,4 \%)$ & $2(5,3 \%)$ & $2(5,3 \%)$ & $5(13,2 \%)$ & $18(47,4 \%)$ \\
\hline 15 & $4(10,5 \%)$ & $7(18,4 \%)$ & $3(7,9 \%)$ & $1(2,6 \%)$ & $6(15,8 \%)$ & $17(44,7 \%)$ \\
\hline 16 & $3(7,9 \%)$ & $7(18,4 \%)$ & $1(2,6 \%)$ & $3(7,9 \%)$ & $4(10,5 \%)$ & $20(52,6 \%)$ \\
\hline
\end{tabular}




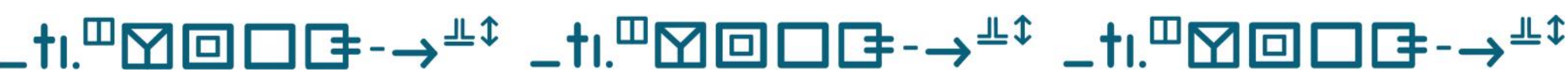

Senso de autoeficácia de professores de alunos com autismo surdos em escolas bilíngues Raquel Aparecida Lopes • Cibelle Albuquerque de La Higuera Amato et al.

\begin{tabular}{|c|c|c|c|c|c|c|}
\hline 17 & $5(13,2 \%)$ & $7(18,4 \%)$ & $0(0,0 \%)$ & $2(5,3 \%)$ & $4(10,5 \%)$ & $20(52,6 \%)$ \\
\hline 18 & $2(5,3 \%)$ & $9(23,7 \%)$ & $1(2,6 \%)$ & $2(5,3 \%)$ & $3(7,9 \%)$ & $21(55,3 \%)$ \\
\hline 19 & $0(0,0 \%)$ & $9(23,7 \%)$ & $0(0,0 \%)$ & $4(10,5 \%)$ & $4(10,5 \%)$ & $21(55,3 \%)$ \\
\hline 20 & $1(2,6 \%)$ & $9(23,7 \%)$ & $1(2,6 \%)$ & $9(23,7 \%)$ & $5(13,2 \%)$ & $13(34,2 \%)$ \\
\hline 21 & $3(7,9 \%)$ & $7(18,4 \%)$ & $1(2,6 \%)$ & $8(21,1 \%)$ & $4(10,5 \%)$ & $15(39,5 \%)$ \\
\hline 22 & $4(10,5 \%)$ & $7(18,4 \%)$ & $2(5,3 \%)$ & $4(10,5 \%)$ & $6(15,8 \%)$ & $15(39,5 \%)$ \\
\hline 23 & $2(5,3 \%)$ & $9(23,7 \%)$ & $0(0,0 \%)$ & $4(10,5 \%)$ & $7(18,4 \%)$ & $16(42,1 \%)$ \\
\hline 24 & $2(5,3 \%)$ & $9(23,7 \%)$ & $1(2,6 \%)$ & $4(10,5 \%)$ & $7(18,4 \%)$ & $15(39,5 \%)$ \\
\hline 25 & $3(7,9 \%)$ & $9(23,7 \%)$ & $1(2,6 \%)$ & $2(5,3 \%)$ & $6(15,8 \%)$ & $17(44,7 \%)$ \\
\hline 26 & $2(5,3 \%)$ & $9(23,7 \%)$ & $3(7,9 \%)$ & $5(13,2 \%)$ & $5(13,2 \%)$ & $14(36,8 \%)$ \\
\hline 27 & $2(5,3 \%)$ & $11(28,9 \%)$ & $3(7,9 \%)$ & $5(13,2 \%)$ & $9(23,7 \%)$ & $8(21,1 \%)$ \\
\hline 28 & $2(5,3 \%)$ & $11(28,9 \%)$ & $3(7,9 \%)$ & $3(7,9 \%)$ & $8(21,1 \%)$ & $11(28,9 \%)$ \\
\hline 29 & $4(10,5 \%)$ & $8(21,1 \%)$ & $4(10,5 \%)$ & $2(5,3 \%)$ & $8(21,1 \%)$ & $12(31,6 \%)$ \\
\hline 30 & $4(10,5 \%)$ & $6(15,8 \%)$ & $7(18,4 \%)$ & $5(13,2 \%)$ & $9(23,7 \%)$ & $7(18,4 \%)$ \\
\hline
\end{tabular}

Fonte: Elaborado pelas autoras (2021).

Descrição da imagem: Tabela informativa, elaborada pelas autoras, que apresenta as respostas dadas pelos professores relacionadas às frequências absoluta e relativa das respostas da escala Autism Self-Efficacy Scale for Teachers (ASSET).

É possível observar a frequência, tanto absoluta quanto relativa, das respostas dadas à escala ASSET. Considerando-se a hipótese de um item receber a mesma porcentagem nas seis respostas possíveis, teríamos 16\% de frequência relativa em cada coluna. Nesta perspectiva, uma frequência alta teria o triplo da frequência média, isto é, 48\%. Assim, têm-se oito respostas nas quais a escala de confiança 5 (certamente posso fazer) se destaca. São elas a 2, 6, 7, 8, 16, 17, 18 e 19, respectivamente: descrever as características do aluno que se relacionam ao autismo; escrever um plano de ensino para o aluno baseado em metas e objetivos; elaborar atividades de 


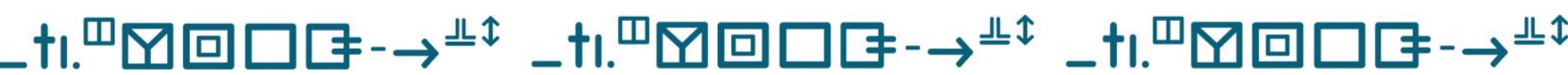

Senso de autoeficácia de professores de alunos com autismo surdos em escolas bilíngues Raquel Aparecida Lopes • Cibelle Albuquerque de La Higuera Amato et al.

ensino para o aluno; organizar a sala de aula para aumentar as oportunidades de aprendizagem para o aluno; reunir informações para avaliar o progresso do aluno em relação aos objetivos propostos; fazer uso dos dados coletados para reavaliar as metas e os objetivos do aluno; avaliar as habilidades de interação social do aluno; e avaliar a habilidade de brincar do aluno.

Percebe-se o alto grau de confiança dos professores neste estudo, o que é um aspecto importante, uma vez que a percepção positiva em relação ao trabalho com seu aluno com TEA é fator crucial para o sucesso do processo de escolarização. A autoeficácia exerce um papel determinante do pensamento, comportamento e desempenho de uma ação, e pode ser desenvolvida partindo-se de quatro fontes de influência: experiências, realizações e desempenhos anteriores, experiência vicariante, persuasão social e estados fisiológicos e afetivos (BANDURA, 1986, 1997). Para o autor, as experiências, realizações e desempenhos anteriores são os fatores mais importantes do desenvolvimento das crenças de autoeficácia, uma vez que a própria experiência se constitui como principal meio de informação sobre as capacidades de uma pessoa. A experiência vicariante parte do princípio de que o que se aprende a partir da experiência do outro também pode influenciar como as pessoas julgam suas habilidades, uma vez que o desempenho do outro fornece informações relevantes sobre quais desempenhos podemos realizar. A persuasão social relaciona-se às respostas avaliativas que o indivíduo recebe do outro sobre suas capacidades para desempenhar uma ação. Os estados fisiológicos e afetivos ocupam um papel mais restrito sobre a autoeficácia, por constituírem um fator situacional que envolvem as respostas físicas e emocionais frente à fatores estressantes, interferindo no julgamento que fazemos de nós mesmos. Indivíduos com alto senso de autoeficácia podem identificar nessas 


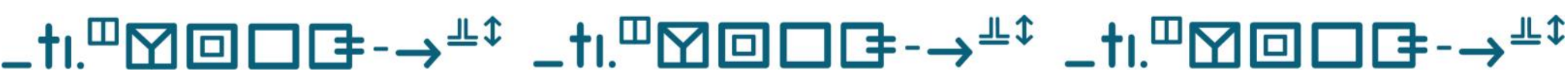

Senso de autoeficácia de professores de alunos com autismo surdos em escolas bilíngues Raquel Aparecida Lopes • Cibelle Albuquerque de La Higuera Amato et al.

respostas componentes facilitadores de sua ação, por outro lado, indivíduos com baixo senso podem identificá-las como fator prejudicial, certamente, um estado emocional positivo favorece o senso de autoeficácia. Em partes, indivíduos contam com informações sobre seu estado fisiológico e afetivo, no julgamento de suas capacidades (BANDURA, 1986, 1993, 1997, SOUZA; BRITO, 2008).

Retomando as análises, é interessante notar que as frequências em destaque não estão concentradas todas no mesmo tipo de item. Há desde entender as características do aluno autista, planejar ensino e atividades e organizar o ambiente, até coletar dados e avaliar o progresso do aluno. Não há, entretanto, um agrupamento de itens que tenha a mesma frequência de respostas em todos os subitens.

Outra forma de observar as respostas é por meio do Gráfico 1, que divide as respostas em dois grupos: os tons em vermelho para as respostas 0,1 e 2 e os tons verdes para as respostas 3, 4 e 5. Os dados utilizados foram as frequências relativas das respostas (\%). Os itens da escala ASSET foram listados em ordem crescente da soma das frequências 3, 4 e 5. Assim, podese visualizar de cima para baixo, dos itens com menos graus de confiança aos itens com maior grau de confiança, conforme agrupamento acima exposto. 


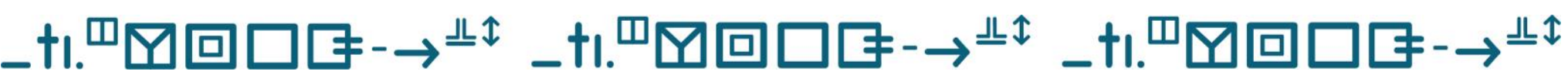

Senso de autoeficácia de professores de alunos com autismo surdos em escolas bilíngues Raquel Aparecida Lopes • Cibelle Albuquerque de La Higuera Amato et al.

Gráfico 1 - Grau de Confiança: Escala ASSET.

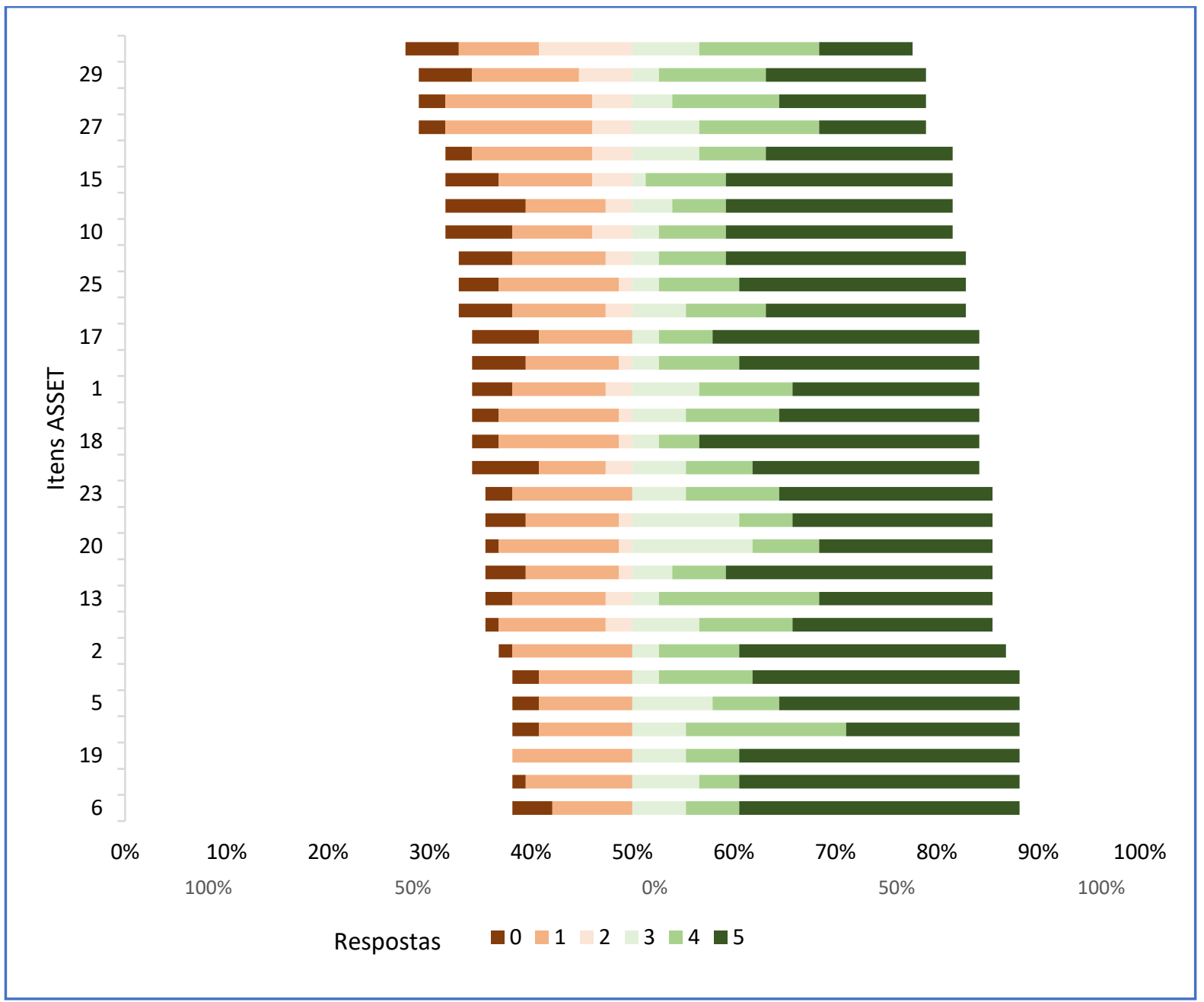

Fonte: Elaborado pelas autoras (2021).

Descrição da imagem: Gráfico informativo, elaborado pelas autoras, que apresenta a prevalência das respostas dadas pelos professores relacionados ao grau de confiança da escala Autism Self-Efficacy Scale for Teachers (ASSET).

Observa-se no Gráfico 1 a prevalência de respostas nos graus de confiança 3, 4 e 5. Apesar das diferentes frequências, os itens 4, 5, 6, 7, 8 e 19 da escala ASSET receberam $76 \%$ das respostas nos graus de maior confiança, respectivamente: interpretar informações da avaliação para definição das metas e dos objetivos de ensino para o aluno; escrever objetivos que possam ser verificados claramente quando atingidos pelo aluno; escrever um plano de ensino para o aluno baseado em metas e 


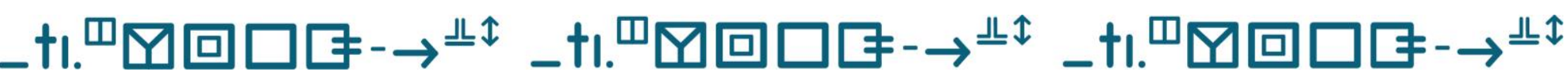

Senso de autoeficácia de professores de alunos com autismo surdos em escolas bilíngues Raquel Aparecida Lopes • Cibelle Albuquerque de La Higuera Amato et al.

objetivos; elaborar atividades de ensino para o aluno; organizar a sala de aula para aumentar as oportunidades de aprendizagem para o aluno; e avaliar a habilidade de brincar do aluno. Os itens 6, 7, 8, e 19 já haviam se destacado entre os que tiveram frequência alta. Há 4 itens nos quais o grau de confiança foi bastante elevado nesta amostragem. As maiores incidências de respostas nos graus 0,1 e 2 ficaram nos itens 27, 28, 29 e 30, recebendo mais de $40 \%$ de frequência de respostas, concomitantemente: sustentar a atenção do aluno; motivar o aluno; auxiliar o aluno a sentir-se competente; e ensinar habilidades acadêmicas ao aluno.

Uma outra forma de organizar os dados coletados é agrupar as respostas 0-1, 2-3 e 4-5. Assim, é possível analisar as respostas em três grupos: pouca confiança, confiança média, muita confiança, conforme Tabela 2. Como as escalas likert com muitos itens possuem pouca frequência de respostas em seus extremos, ao agrupar as respostas, consegue-se ver que, embora oito respostas tenham recebido grau de confiança 5, a maioria das respostas foram nas opções 4 e 5 .

Tabela 2 - Agrupamento das frequências absoluta e relativa das respostas à escala ASSET.

\begin{tabular}{|r|l|l|l|}
\hline \multicolumn{5}{|l|}{$n i(f i)$} \\
\hline Item & Pouca Confiança (0-1) & Confiança Média (2-3) & Muita Confiança (4-5) \\
\hline $\mathbf{1}$ & $10(26,3 \%)$ & $7(18,4 \%)$ & $21(55,3 \%)$ \\
\hline $\mathbf{2}$ & $10(26,3 \%)$ & $2(5,3 \%)$ & $26(68,4 \%)$ \\
\hline $\mathbf{3}$ & $9(23,7 \%)$ & $7(18,4 \%)$ & $22(57,9 \%)$ \\
\hline $\mathbf{4}$ & $9(23,7 \%)$ & $4(10,5 \%)$ & $25(65,8 \%)$ \\
\hline $\mathbf{5}$ & $9(23,7 \%)$ & $6(15,8 \%)$ & $23(60,5 \%)$ \\
\hline $\mathbf{6}$ & $9(23,7 \%)$ & $4(10,5 \%)$ & $25(65,8 \%)$ \\
\hline $\mathbf{7}$ & $9(23,7 \%)$ & $5(13,2 \%)$ & $24(63,2 \%)$ \\
\hline $\mathbf{8}$ & $9(23,7 \%)$ & $2(5,3 \%)$ & $27(71,1 \%)$ \\
\hline
\end{tabular}




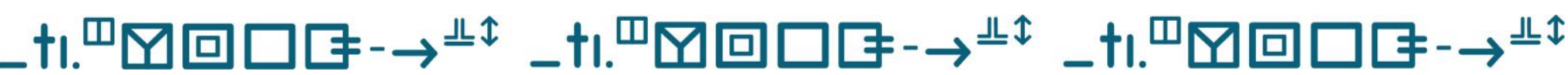

Senso de autoeficácia de professores de alunos com autismo surdos em escolas bilíngues Raquel Aparecida Lopes • Cibelle Albuquerque de La Higuera Amato et al.

\begin{tabular}{|c|c|c|c|}
\hline 9 & $11(28,9 \%)$ & $3(7,9 \%)$ & $24(63,2 \%)$ \\
\hline 10 & $11(28,9 \%)$ & $5(13,2 \%)$ & $22(57,9 \%)$ \\
\hline 11 & $12(31,6 \%)$ & $5(13,2 \%)$ & $21(55,3 \%)$ \\
\hline 12 & $10(26,3 \%)$ & $6(15,8 \%)$ & $22(57,9 \%)$ \\
\hline 13 & $9(23,7 \%)$ & $4(10,5 \%)$ & $25(65,8 \%)$ \\
\hline 14 & $11(28,9 \%)$ & $4(10,5 \%)$ & $23(60,5 \%)$ \\
\hline 15 & $11(28,9 \%)$ & $4(10,5 \%)$ & $23(60,5 \%)$ \\
\hline 16 & $10(26,3 \%)$ & $4(10,5 \%)$ & $24(63,2 \%)$ \\
\hline 17 & $12(31,6 \%)$ & $2(5,3 \%)$ & $24(63,2 \%)$ \\
\hline 18 & $11(28,9 \%)$ & $3(7,9 \%)$ & $24(63,2 \%)$ \\
\hline 19 & $9(23,7 \%)$ & $4(10,5 \%)$ & $25(65,8 \%)$ \\
\hline 20 & $10(26,3 \%)$ & $10(26,3 \%)$ & $18(47,4 \%)$ \\
\hline 21 & $10(26,3 \%)$ & $9(23,7 \%)$ & $19(50,0 \%)$ \\
\hline 22 & $11(28,9 \%)$ & $6(15,8 \%)$ & $21(55,3 \%)$ \\
\hline 23 & $11(28,9 \%)$ & $4(10,5 \%)$ & $23(60,5 \%)$ \\
\hline 24 & $11(28,9 \%)$ & $5(13,2 \%)$ & $22(57,9 \%)$ \\
\hline 25 & $12(31,6 \%)$ & $3(7,9 \%)$ & $23(60,5 \%)$ \\
\hline 26 & $11(28,9 \%)$ & $8(21,1 \%)$ & $19(50,0 \%)$ \\
\hline 27 & $13(34,2 \%)$ & $8(21,1 \%)$ & $17(44,7 \%)$ \\
\hline 28 & $13(34,2 \%)$ & $6(15,8 \%)$ & $19(50,0 \%)$ \\
\hline 29 & $12(31,6 \%)$ & $6(15,8 \%)$ & $20(52,6 \%)$ \\
\hline 30 & $10(26,3 \%)$ & $12(31,6 \%)$ & $16(42,1 \%)$ \\
\hline
\end{tabular}

$n=38$

Fonte: Elaborado pelas autoras (2021).

Descrição da imagem: Tabela informativa, elaborada pelas autoras, que apresenta as respostas dadas pelos professores relacionadas ao agrupamento das frequências absoluta e relativa das respostas à escala Autism Self-Efficacy Scale for Teachers (ASSET).

Verificando o agrupamento, destacam-se as respostas que somam mais de um terço das respostas. Para o agrupamento de pouca confiança, percebe-se que, embora não seja o agrupamento com mais respostas, os itens 27 e 28 (sustentar a atenção do aluno e motivar o aluno) receberam 


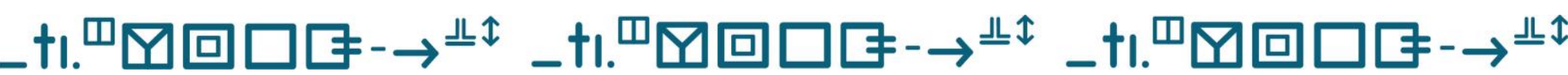

Senso de autoeficácia de professores de alunos com autismo surdos em escolas bilíngues Raquel Aparecida Lopes • Cibelle Albuquerque de La Higuera Amato et al.

pouco mais de um terço das respostas. Assim, pode-se notar que sustentar a atenção do aluno e motivar o aluno são itens que merecem destaque e podem ser explicados pelos déficits presentes na habilidade de atenção compartilhada, associados aos processos afetivos, cognitivos e motivacionais atípicos presentes no desenvolvimento de crianças com TEA (MUNDY; CROWSON, 1997; TOMASELLO, 2003; OSÓRIO et al., 2011). Para os autores a atenção compartilhada é a habilidade de coordenar o foco entre dois parceiros sociais em relação a um terceiro referencial externo com a intenção de partilhar uma experiência comum. Existem dois tipos de comportamento de atenção compartilhada, o primeiro refere-se à iniciação da atenção compartilhada, que são as tentativas espontâneas das crianças em direcionar a atenção do adulto, e o segundo é a resposta à atenção compartilhada, que está relacionada às respostas da criança em relação às sugestões do outro (MUNDY; CROWSON, 1997; OSÓRIO et al., 2011).

É possível observar na Tabela 2, que embora o agrupamento de muita confiança seja sempre o agrupamento com maior frequência de respostas, o agrupamento de pouca confiança possui maior frequência que o agrupamento de confiança média. As respostas estavam frequentemente nos extremos. Ou há nada para se fazer ou pode se fazer algo com certeza. Mesmo não havendo um item central, as respostas 2 e 3 e foram menos escolhidas que as respostas 1 e 2.

Dois itens fogem da frequência observada. O item 20 (ensinar habilidades/estratégias de interação social ao aluno) empatou entre os agrupamentos de pouca confiança e confiança média; e o item 30 (ensinar habilidades acadêmicas ao aluno) teve o agrupamento de confiança média com pouco maior de frequência que o agrupamento de pouca confiança. Pode-se afirmar que estes dois itens receberam de forma mais equilibrada as respostas. Estas frequências equilibradas revelam não apenas um 


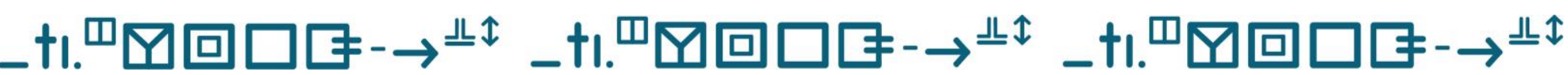

Senso de autoeficácia de professores de alunos com autismo surdos em escolas bilíngues Raquel Aparecida Lopes • Cibelle Albuquerque de La Higuera Amato et al.

equilíbrio das respostas, mas podem conter outros fatores, como o entendimento da pergunta pelos professores. As habilidades sociais são comportamentos com grande probabilidade de causar consequências reforçadoras para o indivíduo e para as demais pessoas do grupo social (DEL PRETTE; DEL PRETTE, 2010). Há evidências substanciais de comprometimento das habilidades de interação social em crianças com autismo e sabendo que as habilidades são aprendidas na interação com o outro, se tornam extremamente necessários promover programas educacionais estruturados e intervenções planejadas com o intuito de criar condições de aprendizagem no ambiente escolar.

O estudo de Freitas e Del Prette (2013) analisou 12 categorias de necessidades especiais, e os resultados de escores mais baixos de habilidades sociais foram: Autismo, TDAH e problemas de comportamento internalizantes e externalizantes. Tais categorias englobam prejuízo ao desenvolvimento social infantil, o que provavelmente influencia também demais áreas do desenvolvimento. No que se refere às crianças surdas, o estudo revelou que apresentam um repertório de habilidades sociais mediano em comparação com as categorias citadas e escores mais homogêneos nas diferentes classes de habilidades sociais.

O cenário nacional educacional sugere a urgência de estudos sobre habilidades sociais em crianças com TEA surdas tanto em ambientes bilíngues como em escolas inclusivas.

\section{Conclusão}

A percepção de autoeficácia docente em relação à prática profissional pode afetar a motivação e a atuação do professor frente às atitudes pedagógicas e influenciar diretamente no processo escolar de alunos com 


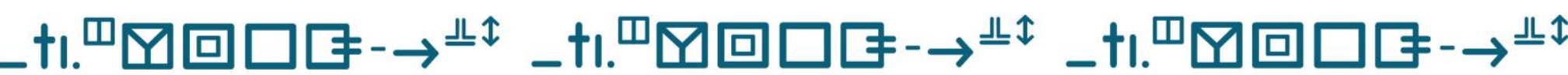

Senso de autoeficácia de professores de alunos com autismo surdos em escolas bilíngues Raquel Aparecida Lopes • Cibelle Albuquerque de La Higuera Amato et al.

TEA. O estudo objetivou identificar e analisar o senso de autoeficácia docente (AED) de professores de alunos com TEA surdos em espaços escolares bilíngues. Conforme mencionado anteriormente, não existem escalas no Brasil que avaliam a autoeficácia no desempenho das funções de professor no que se refere à atendimento de alunos com TEA surdos. Neste caso, a ASSET mostrou-se um importante instrumento de autoavaliação dos profissionais investigados neste estudo.

Certamente que escalas de autoavaliação carregam uma enorme carga subjetiva em suas respostas. Entretanto, considera-se que os objetivos propostos nesta investigação foram alcançados, uma vez que a análise do corpus revelou uma amostra de respostas que ajuda a pensar sobre como o professor vê suas práticas profissionais em relação ao ensino dos alunos. A maior parte da amostragem está confiante com todos os itens avaliados. Isto pode significar maior preparo dos docentes (a maioria dos profissionais que responderam ao questionário possui pós-graduação lato sensu), mais informação disponível, maior apoio das famílias e instituições. Todos estes fatores, aliados com fatores mais profundos, como uma mudança cultural e da forma de pensar a educação especial, certamente possuem influência nestes resultados. Obviamente que o intuito do estudo não foi discutir todos estes pontos.

Não houve a intenção em dividir a amostragem em grupos e analisar as respostas de grupos por gênero, formação, localidade, tempo de atuação. Uma continuação dessas análises ajudaria entender melhor o ensino de autistas surdos no contexto atual da escola bilingue.

Os resultados aqui apresentados levam a refletir sobre modelos de formação continuada que, sem dúvida, estão intrinsicamente relacionados com a motivação, realização profissional e interesse pelas práticas pedagógicas executadas cotidianamente. Dito isso, poderíamos nos 


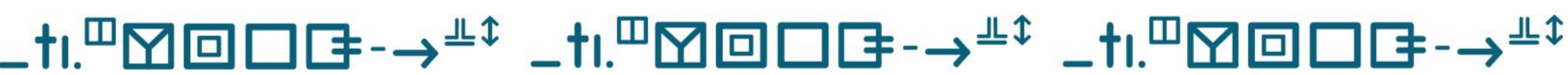

Senso de autoeficácia de professores de alunos com autismo surdos em escolas bilíngues Raquel Aparecida Lopes • Cibelle Albuquerque de La Higuera Amato et al.

questionar também se as concepções e práticas pedagógicas dos participantes investigados neste estudo são sustentadas em evidências científicas, uma vez que a literatura aponta que, mesmo que professores possuam um conhecimento sobre a etiologia dos TEA, há um percentual, mesmo que baixo com alguns conceitos errôneos, ainda que tenham boa formação em nível de graduação e pós-graduação e experiência com alunos com TEA ao longo da sua trajetória profissional. Dentre estes conceitos, podemos relacionar o baixo conhecimento sobre a etiologia do TEA, adaptação curricular, manejo comportamental e estimulação de habilidades de aprendizagens, o que revela o despreparo destes profissionais no Brasil.

Frente a este fato, as propostas de formação docente para o trabalho com alunos com TEA surdos devem estar baseadas na relação teoria e prática, e pensadas nas especificidades de cada um por diferentes perspectivas. Apostar em formação continuada possivelmente resultaria em um elevado senso de autoeficácia e melhoria no desempenho das funções docentes, fazendo com que estes profissionais superem os desafios atuais que a educação bilingue no Brasil apresenta.

\section{Referências}

AMERICAN PSYCHIATRIC ASSOCIATION. Manual Diagnóstico e Estatístico de Transtornos Mentais-DSM-5. Porto Alegre: Artes Médicas, 2014.

ARAÚJO, C. A. Psicologia e os Transtornos do Espectro do Autismo. In.: SCHWARTZMAN, J. S.; ARAÚJO, C. A. (Org.). Transtorno do Espectro do Autismo. São Paulo: Memnon, 2011. p.173-201.

AZZI, R. G.; POLYDORO, S. A. J. Autoeficácia proposta por Albert Bandura: Algumas discussões. In: Autoeficácia em diferentes contextos. Campinas: Alinea, 2006. p. 9-23. 


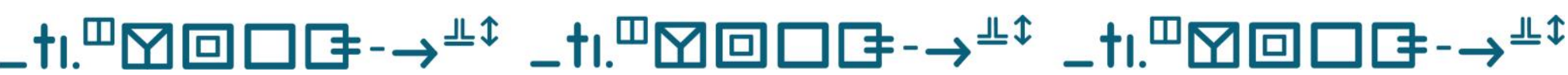

Senso de autoeficácia de professores de alunos com autismo surdos em escolas bilíngues Raquel Aparecida Lopes • Cibelle Albuquerque de La Higuera Amato et al.

BANDURA, A. Social foundations of thought and action: a social cognitive theory. Englewood Cliffs, N. J., Prentice Hall, 1986.

Perceived self-efficacy in cognitive development and functioning. Educational Psychologist, v. 28, n. 2, p. 117-148, 1993.

. Self-efficacy: the exercise of control. New York: W. H. Freeman, 1997. BRASIL. Política Nacional de Educação Especial na Perspectiva da Educação Inclusiva. Brasília: MEC/SECADI, 2008. Disponível em: <http://portal.mec.gov.br/arquivos/pdf/politicaeducespecial.pdf.>. Acesso em: 23 maio 2021.

BROUWERS, A.; TOMIC, W. O The factorial validity of scores on the teacher interpersonal self-efficacy scale. Educational and Psychological Measurement. v. 61, n. 3, p. 432-445, 2001.

BZUNECK, J. A.; GUIMARÃES, S. É. R. Crenças de eficácia de professores: validação da escala de Woolfolk e Hoy. Psico-USF, v. 8, n. 2, p. 137-143, 2003. CAMPOS, S. R. L. A representação social dos professores de surdos sobre o ensino de Libras e Língua Portuguesa no Ensino Fundamental I. 2017. 280 p. Tese (Doutorado em Educação) - Faculdade de Educação. Universidade de São Paulo, São Paulo, 2017.

CANABARRO, R. C. C.; TEIXEIRA, M. C. T. V.; SHIMIDT, C. Tradução e adaptação transcultural da escala de avaliação de autoeficácia de professores de alunos com autismo: autism self-efficacy scale for teachers (ASSET). Revista Brasileira de Educação Especial, v. 24, n. 2, p. 229-246, 2018.

CARR, E. G. et al. Acquisition of signlanguage by autistic children. I: Expressive labelling. Journal of Applied Behavior Analysis, v. 11, n. 4, p. 489-501, 1978. DEL PReTTE, Z. A. P.; DEL PRETTE, A. Habilidades sociais e análise do comportamento: Proximidade histórica e atualidades. Revista Perspectivas, v. 1, n. 2, p. 104-115, 2010. 


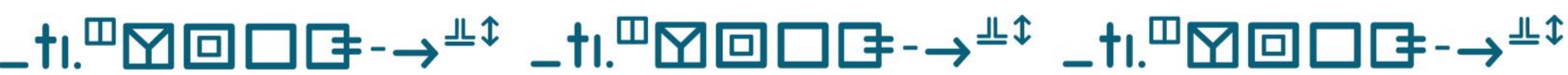

Senso de autoeficácia de professores de alunos com autismo surdos em escolas bilíngues Raquel Aparecida Lopes • Cibelle Albuquerque de La Higuera Amato et al.

FELIPE, T. A. Libras em Contexto. Brasília: MEC/SEESP, 2007. Disponível em: https://pt.slideshare.net/Jorgee_big/libras-em-contexto.>. Acesso em: 24 maio 2021.

FREITAS, L. C.; DEL PRETTE, Z. A. P. Habilidades sociais de crianças com diferentes necessidades educacionais especiais: Avaliação e implicações para intervenção. Avances en Psicología Latinoamericana, v. 31, n. 2, p. 344-362, 2013.

FULWILER, R. L.; FOUTS, T. S. Acquisition American sign language by a noncommunicating autistic child. Journal of Autism and Childbood Schizophrenia, v. 6, n. 1, p. 43-51, 1976.

GARRUTTI-LOURENÇO, E. A. Bilinguismo para surdos e inclusão escolar: a busca por um caminhar articulado. In: MARTINS, E.; CÂNDIDO, R.M. Na trilha da inclusão: deficiência, diferença e desigualdade na escola. São Paulo: Alameda Casa Editorial, 2017.

GIBSON, S.; DEMBO, M. Teacher efficacy: A construct validation. Journal of Educational Psychology, v. 76, n. 4, p. 569-582, 1984.

HARRISON, K. M. P.; MOURA, M. C. A Escola para surdo - escolas em um novo contexto. In: CAMPIOTTO, A.R. et al. (Org.). Novo tratado de fonoaudiologia. São Paulo: Manole, 2013.

KLASSEN, R. et al. Teacher efficacy research 1998-2009: Signs of progress or unfulfilled promise? Educational Psychology Review, v. 23, n. 1, p. 21-43, 2011.

LOPES, R. A. Libras na formação inicial de professores. Belo Horizonte: Editora Dialética, 2020.

Autismo e Surdez: uma análise das estratégias de comunicação e autoeficácia docente em escolas bilíngues para Surdos. 2021. 166 p. Tese. (Doutorado em Distúrbios do Desenvolvimento) - Programa de pós-graduação em 


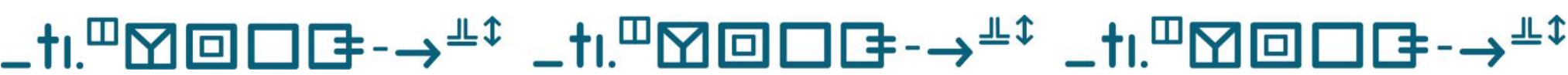

Senso de autoeficácia de professores de alunos com autismo surdos em escolas bilíngues Raquel Aparecida Lopes • Cibelle Albuquerque de La Higuera Amato et al.

Distúrbios do Desenvolvimento. Universidade Presbiteriana Mackenzie, São Paulo, 2020.

LOPES, R. A.; AMATO, C. A. H. A utilização de libras no ambiente escolar para crianças com transtorno do espectro do autismo surdas. In: PIMENTAL, B. N. (Org.). Fundamentos Científicos e prática clínica em fonoaudiologia. Ponta Grossa: Atena, 2021. p. 119-134.

LOPES, R. A.; AMATO, C. A. H. Libras \& Autismo: um diálogo possível?. Belo Horizonte: Editora Dialética, 2021.

LUZ, M. H. S; GOMES, C. A.; LIRA, A. Narrativas sobre a inclusão de uma criança autista: desafios à prática docente. Educación, v. XXVI, n. 50, 2017. Disponível em: $\quad$ <http://www.scielo.org.pe/scielo.php?pid=S101994032017000100007\&script=sci_abstract. $>$

Acesso em: 24 maio 2021.

MOURA, M. C. Libras e Fonoaudiologia: territórios a serem compartilhados. In: ARAÚJO, A. N. et al. (Org.). Questões contemporâneas da clínica fonoaudiológica. São Paulo: Pulso. 2018.

O Surdo: caminhos para uma Nova Identidade. Rio de Janeiro: Revinter, 2000.

Surdez e linguagem. In: FEITOSA, C.B.S.L.; FERREIRA, L. (Org.). Tenho um aluno surdo, e agora? Introdução à libras e educação de surdos. São Carlos: Edufscar, 2013. p. 13-26.

MUNDY, P.; CROWSON, M. Joint attention and early social communication: implications for research on intervention with autism. Journal of Autism and Developmental Disorders, v 27, n. 6, p. 653-76,1997.

NUNES, D. R. P; AZEVEDO, M. Q. O.; SCHMIDT, C. Inclusão educacional de pessoas com autismo no Brasil: uma revisão de literatura. Revista de Educação Especial, v. 26, n. 47, p. 557-572, 2013. 


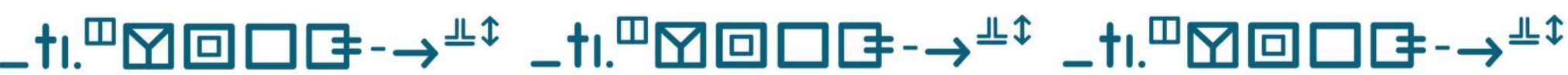

Senso de autoeficácia de professores de alunos com autismo surdos em escolas bilíngues Raquel Aparecida Lopes • Cibelle Albuquerque de La Higuera Amato et al.

OSÓRIO, A. et al. Individual and relational contributions to parallel and joint attention in infancy. Infant Behavior and Development, v. 34, n. 4, p. 515524, 2011.

PAJARES, F.; OLAZ, F. Teoria social cognitiva e autoeficácia: uma visão geral. In: BANDURA, A. et al. (Org). Teoria social cognitiva: conceitos básicos. Porto Alegre: Artmed, 2008. p. 97-114.

RUBLE, L. et al. Preliminary Study of the Autism Self-Efficacy Scale for Teachers (ASSET). Research in Autism Spectrum Disorders, v. 7, n. 9, p. 1151-1159, 2013.

SHIELD, A.; MEIER R. P.; FLUSBERG, H. T. The use of sign language pronouns by native-signing children. with autism. Journal of Autism and Developmental Disorders, v. 45, p. 2128-2145, 2015.

SOUZA, L. F. N. I.; BRITO, M. R. F. Crenças de autoeficácia, autoconceito e desempenho em matemática. Estudos de Psicologia, v. 25, n. 2, p. 193-201, 2008.

STULL, S. et al. Sign language as a means of communicating with autistic and mentally handicapped children. Child and Youth Care Forum, v.8, n.2, p. 143-147, 1979.

TOMASELLO, M. Origens culturais da aquisição do conhecimento humano. São Paulo: Martins Fontes, 2003.

\section{Publisher}

UNIVERSIDADE FEDERAL DE GOIÁS. CURSOS DE LETRAS: LIBRAS E DE LETRAS: TRADUÇÃO E INTERPRETAÇÃO EM LIBRAS/PORTUGUÊS DA FACUldade de letras/UfG. Publicação no PORTAL DE PERIÓdicos UFG. AS IDEIAS EXPRESSADAS NESTE ARTIGO SÃO DE RESPONSABILIDADE DE SUAS AUTORAS, NÃO REPRESENTANDO, NECESSARIAMENTE, A OPINIÃO DOS EDITORES OU DA UNIVERSIDADE. 
